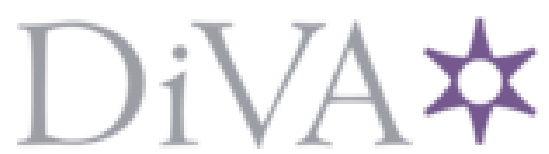

http://www.diva-portal.org

\title{
Postprint
}

This is the accepted version of a paper published in Applied Physics Letters. This paper has been peerreviewed but does not include the final publisher proof-corrections or journal pagination.

Citation for the original published paper (version of record):

Mazraati, H., Chung, S., Houshang, A., Dvornik, M., Piazza, L. et al. (2016)

Low operational current spin Hall nano-oscillators based on NiFe/W bilayers.

Applied Physics Letters, 109(24): 242402

https://doi.org/10.1063/1.4971828

Access to the published version may require subscription.

N.B. When citing this work, cite the original published paper.

Permanent link to this version:

http://urn.kb.se/resolve?urn=urn:nbn:se:kth:diva-201252 


\section{Low operational current spin Hall nano-oscillators based on NiFe/W bilayers}

Hamid Mazraati, Sunjae Chung, Afshin Houshang, Mykola Dvornik, Luca Piazza, Fatjon Qejvanaj, Sheng Jiang, Tuan Q. Le, Jonas Weissenrieder, and Johan Ảkerman

Citation: Appl. Phys. Lett. 109, 242402 (2016); doi: 10.1063/1.4971828

View online: http://dx.doi.org/10.1063/1.4971828

View Table of Contents: http://aip.scitation.org/toc/apl/109/24

Published by the American Institute of Physics

\section{Articles you may be interested in}

Route toward high-speed nano-magnonics provided by pure spin currents

Appl. Phys. Lett. 109, 252401252401 (2016); 10.1063/1.4972244

Large influence of capping layers on tunnel magnetoresistance in magnetic tunnel junctions

Appl. Phys. Lett. 109, 242403242403 (2016); 10.1063/1.4972030

Microwave emission power exceeding $10 \mu \mathrm{W}$ in spin torque vortex oscillator

Appl. Phys. Lett. 109, 252402252402 (2016); 10.1063/1.4972305

Enhancing the injection locking range of spin torque oscillators through mutual coupling Appl. Phys. Lett. 109, 252404252404 (2016); 10.1063/1.4972346

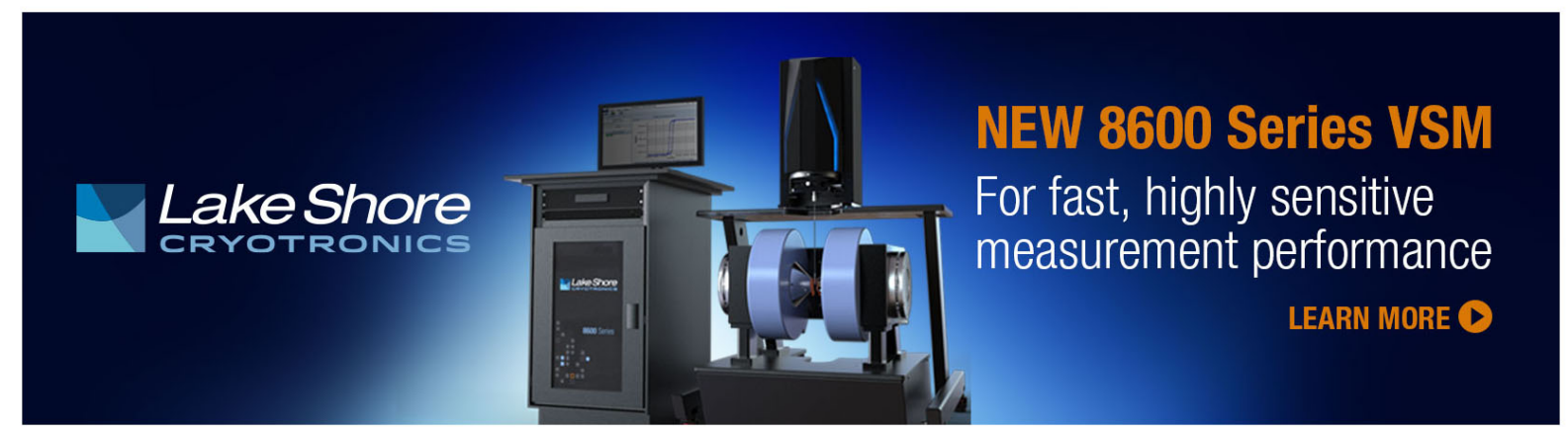




\title{
Low operational current spin Hall nano-oscillators based on NiFe/W bilayers
}

\author{
Hamid Mazraati, ${ }^{1,2, a)}$ Sunjae Chung, ${ }^{2,3, a)}$ Afshin Houshang, ${ }^{1,3}$ Mykola Dvornik, ${ }^{3}$ \\ Luca Piazza, ${ }^{2}$ Fatjon Qejvanaj, ${ }^{1,2}$ Sheng Jiang, ${ }^{1,2}$ Tuan Q. Le, ${ }^{2}$ Jonas Weissenrieder, ${ }^{2}$ \\ and Johan Akerman 1,2,3 \\ ${ }^{1}$ NanOsc AB, Kista 164 40, Sweden \\ ${ }^{2}$ Department of Materials and Nanophysics, School of Information and Communication Technology, \\ KTH Royal Institute of Technology, Electrum 229, SE-16440 Kista, Sweden \\ ${ }^{3}$ Department of Physics, University of Gothenburg, 41296 Gothenburg, Sweden
}

(Received 28 October 2016; accepted 24 November 2016; published online 15 December 2016)

\begin{abstract}
We demonstrate highly efficient spin Hall nano-oscillators (SHNOs) based on NiFe/ $\beta$-W bilayers. Thanks to the very high spin Hall angle of $\beta$-W, we achieve more than a $60 \%$ reduction in the autooscillation threshold current compared to $\mathrm{NiFe} / \mathrm{Pt}$ bilayers. The structural, electrical, and magnetic properties of the bilayers, as well as the microwave signal generation properties of the SHNOs, have been studied in detail. Our results provide a promising path for the realization of low-current SHNO microwave devices with highly efficient spin-orbit torque from $\beta$-W. Published by AIP Publishing.

[http://dx.doi.org/10.1063/1.4971828]
\end{abstract}

Spin Hall nano-oscillators (SHNOs) are promising microwave signal generators with a high degree of frequency tunability, both via the electrical current and the magnitude and direction of an external magnetic field. ${ }^{1}$ These SHNOs operate on the basis of spin-orbit torque (SOT), ${ }^{2}$ which is produced when a spin current, created via the spin Hall $\mathrm{effect}^{3-8}$ in a metal with high spin-orbit coupling (e.g., $\mathrm{Pt},{ }^{9} \mathrm{~W},{ }^{10,11} \mathrm{Ta}^{12}$ ), is absorbed by an adjacent ferromagnetic layer. SOT can act as a negative spin-wave damping in the ferromagnet and above a certain threshold current, it can sustain a steady state autooscillation of the local magnetization.

To date, most SHNOs have been based on $\mathrm{NiFe} /$ $\mathrm{Pt},{ }^{2,13-20} \mathrm{YIG} / \mathrm{Pt}^{21,22}$ and $\mathrm{CoFeB} / \mathrm{Pt}^{23},{ }^{23}$ with SOT produced by a Pt layer. Many other heavy metals, such as $\mathrm{W},{ }^{10,11}$ $\mathrm{IrCu},{ }^{24,25}$ and $\mathrm{CuBi}^{26}{ }^{26}$ have however been investigated, revealing that the so-called spin Hall angle $\left(\theta_{S H}\right)$, which describes the charge current to spin current conversion efficiency, can exceed that of Pt. W is particularly interesting, as it has two different structural phases, called $\alpha$ and $\beta$, with an order of magnitude difference in resistivity $\left(\rho_{\alpha}<\rho_{\beta}\right)$, and a much larger $\theta_{S H}$ in the $\beta$ phase. ${ }^{10,11,27}$ However, $\beta$-W can only be obtained under restricted conditions, such as well-controlled slow deposition rates, no heating, and thin layer thicknesses.

In this study, we demonstrate the fabrication and operation of nano-constriction based SHNOs employing $\beta-\mathrm{W}$ as the heavy metal. We find a large negative value of $\theta_{S H}=-0.385$ and a corresponding dramatic reduction of the SHNO threshold current by over $60 \%$ compared to Pt-based SHNOs. ${ }^{2,13-19,21-23}$

All thin films were deposited at room temperature on cplane sapphire substrates using magnetron sputtering in a 2.5 mTorr Ar atmosphere, in an ultra-high vacuum (base pressure below $1 \times 10^{-8}$ mTorr) AJA Orion 8 sputtering system. First we carried out a detailed study of the thickness dependence of the $\mathrm{W}$ film resistivity in films deposited at a rate of $0.07 \AA / \mathrm{s}$. Figure 1 shows that, as the thickness increases, the

${ }^{a)}$ H. Mazraati and S. Chung contributed equally to this work.
W resistivity decreases exponentially from a very high value $(\sim 400 \mu \Omega \mathrm{cm})$ at a thickness of $3 \mathrm{~nm}$ and finally drops to a near constant value of $\sim 30 \mu \Omega \mathrm{cm}$ for thicknesses of $12 \mathrm{~nm}$ and above, which is close to the bulk $\mathrm{W}$ resistivity. This indicates that films with a thickness below $10 \mathrm{~nm}$ are primarily of $\beta$-phase, and a W thickness of $5 \mathrm{~nm}$ was therefore chosen for the fabrication of SHNO devices.

Prior to the device processing, a bilayer consisting of $5 \mathrm{~nm} \mathrm{Ni}_{80} \mathrm{Fe}_{20}$ (Py) and $5 \mathrm{~nm} \beta$-phase $\mathrm{W}$ was deposited, with the thicknesses predetermined from $\mathrm{X}$-ray reflectivity measurements on calibration films. High-resolution transmission electron microscopy (HR-TEM) (Figure 1-inset) confirms the thicknesses of the bilayer and also shows that the $\mathrm{Py} / \beta$-W interface is well-defined without any significant intermixing. The distance between lattice fringes in the W-layer was found from a fast Fourier transformation to be approximately $2.50 \AA$, i.e., close to the (l 002 ) plane of $\beta$-W. ${ }^{28}$ This confirms that the $\beta$-W crystal structure is indeed realized in the stack.

To fabricate SHNOs, the bilayer was patterned into an array of $4 \mu \mathrm{m} \times 12 \mu \mathrm{m}$ rectangular mesas using photolithography and dry etching. Nano-constrictions with the width

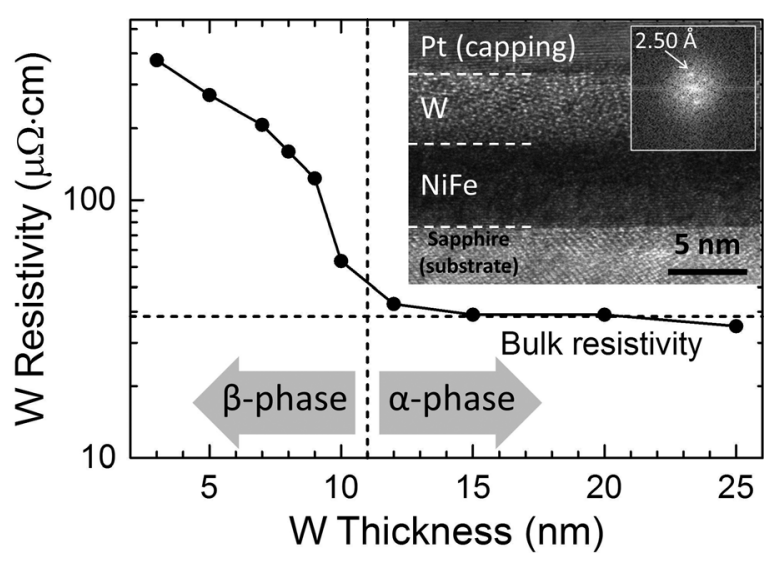

FIG. 1. Resistivity vs. thickness for W thin films. Inset: Cross-section HRTEM image of the stack and the result of fast Fourier transformation of the W-layer. 
varying from 80 to $160 \mathrm{~nm}$ were subsequently fabricated in the center of these mesas by a combination of e-beam lithography and Argon ion milling. To determine $\theta_{S H}$ of tungsten, $6 \mu \mathrm{m}$-wide bars were fabricated simultaneously next to the SHNO mesas, and characterized using spin-torque-induced ferromagnetic resonance (ST-FMR) measurements. Finally, a conventional ground-signal-ground (GSG) waveguide and electrical contact pads for wide frequency range microwave measurement were fabricated using lift-off photolithography and $\mathrm{Cu} / \mathrm{Au}$ sputtering on top of both the SHNO nanoconstrictions and ST-FMR bars.

A schematic of the device structure, an atomic force microscopy (AFM) image, and a scanning electron microscopy (SEM) image of the fabricated $100 \mathrm{~nm}$-wide nano-constriction SHNO device are shown in Figure 2(a), where the direction of the applied in-plane field and current are also defined: the field angle $\phi=0^{\circ}$ along $+x$ and $+90^{\circ}$ along $+y$, while a negative (positive) current means electrons flow along $+(-) y$.

The linear nano-constriction width dependence of the SHNO resistance shown in Figure 2(b) indicates that the device-to-device variation during the fabrication process was moderate. ST-FMR measurements based on homodyne detection were then carried out on $6 \mu \mathrm{m}$ bar-shaped devices, using a $313 \mathrm{~Hz}$-pulse-modulated microwave signal and a $d c$ current injected simultaneously through $r f$ and $d c$ ports, respectively, of a bias-tee. The $d c$ voltage response $\left(V_{d c}\right)$ from the $313 \mathrm{~Hz}$ modulated microwave input is detected also through the $d c$ port of the bias-tee using a standard lock-in amplifier. $^{21,29-32}$ Figures 3(a) and 3(b) show the resonance peaks extracted from the detected ST-FMR output spectra with the in-plane magnetic field $\left(H_{I P}\right)$ swept from 0 to
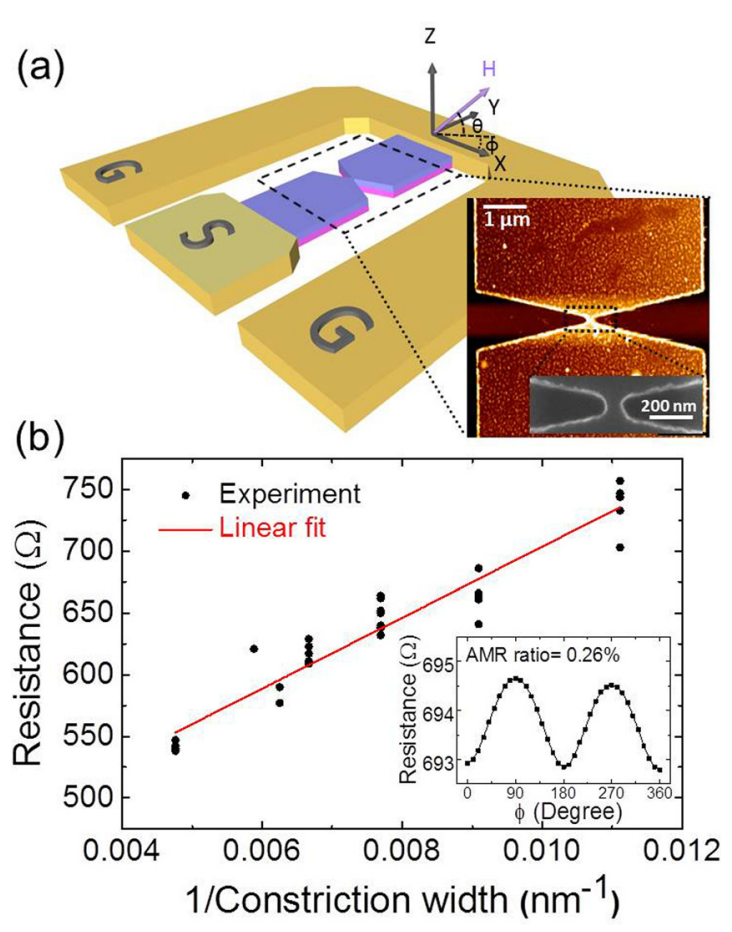

FIG. 2. (a) Schematic of the device structure and the configuration of the applied magnetic field. Inset: AFM image of the active nano-constriction area with zoomed-in SEM image of the nano-constriction. (b) Device resistance vs. constriction width. Inset: AMR measurement for constriction width $=100 \mathrm{~nm}$.
2500 Oe at different constant frequencies $f$ of the injected microwave signal.

Microwave measurements were carried out using our custom-built setup in which a $d c$ current was driven into the SHNO and the output microwave signal was picked up separately via the bias-tee. The output signal was amplified by $35 \mathrm{~dB}$ using a broadband $(0.1-20 \mathrm{GHz})$ low-noise amplifier (LNA) prior to an R\&S FSQ26 spectral analyzer. Results of the microwave measurement in the applied in-plane and outof-plane (OOP) fields are shown in Figures 4 and 5, respectively. All measurements were performed at room temperature.

Figure 3(a) shows the ST-FMR results of a $6 \mu \mathrm{m}$-wide bar-shaped device in an in-plane magnetic field $\left(H_{I P}\right)$ applied along $\phi_{H}=30^{\circ}$ and swept from 0 to $2.5 \mathrm{kOe}$. All ST-FMR spectra have been fitted to a function consisting of one symmetric and one antisymmetric Lorentzian having the same resonance field and linewidth ${ }^{9}$

$$
V_{d c}=\frac{V_{S} \Delta H^{2}+4 V_{A}\left(H_{I P}-H_{r}\right) \Delta H}{4\left(H_{I P}-H_{r}\right)^{2}+\Delta H^{2}}+V_{\mathrm{offset}},
$$

where $H_{r}$ is the resonance field of the measurement spectra, $\Delta H$ is its linewidth (full-width half maximum, FWHM), $V_{S}$ and $V_{A}$ are the coefficients of the symmetric and antisymmetric Lorentzian functions, respectively, and $V_{\text {offset }}$ is a constant offset voltage.

The color plot of the ST-FMR spectra is shown in Figure 3(a). The dependence of the resonance field $\left(H_{r}\right)$ on the microwave frequency shows good agreement with the Kittel formula (solid red line), ${ }^{33}$ and by considering the gyromagnetic ratio of $\gamma / 2 \pi=28 \mathrm{GHz} / \mathrm{T}$, the extracted effective magnetization from the fit is $\mu_{0} M_{\text {eff }}=0.71 \mathrm{~T}$. The inset in Figure 3(a) shows the
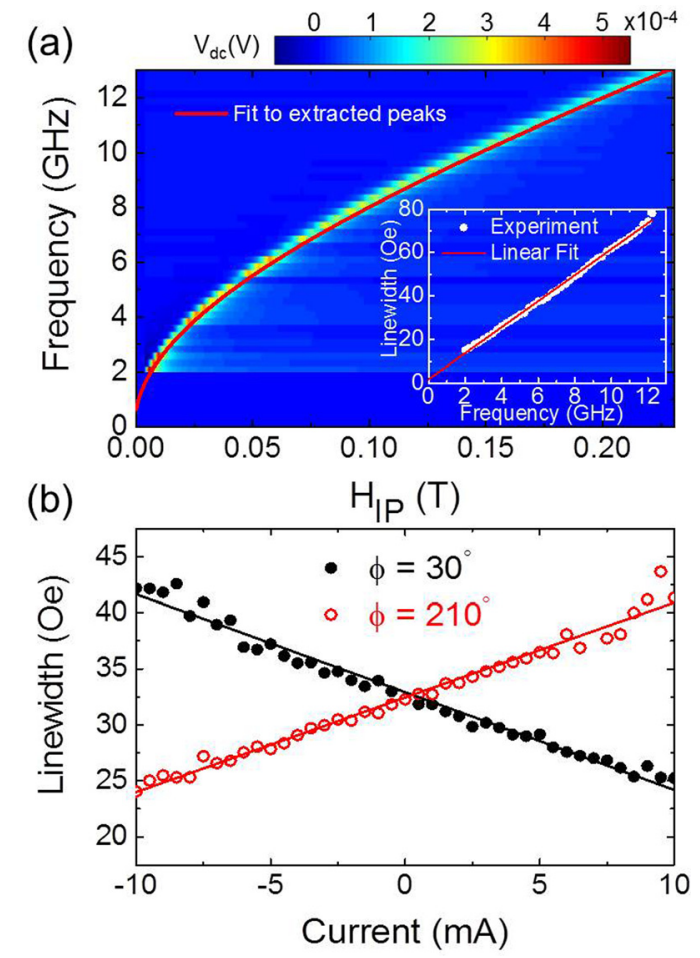

FIG. 3. (a) ST-FMR measurement on a $6 \mu \mathrm{m}$-wide bar-shaped structure. Inset: ST-FMR linewidth vs. frequency. (b) ST-FMR linewidth vs. current in an inplane magnetic field along $\phi=30^{\circ}$ (black dots) and $210^{\circ}$ (red dots). 


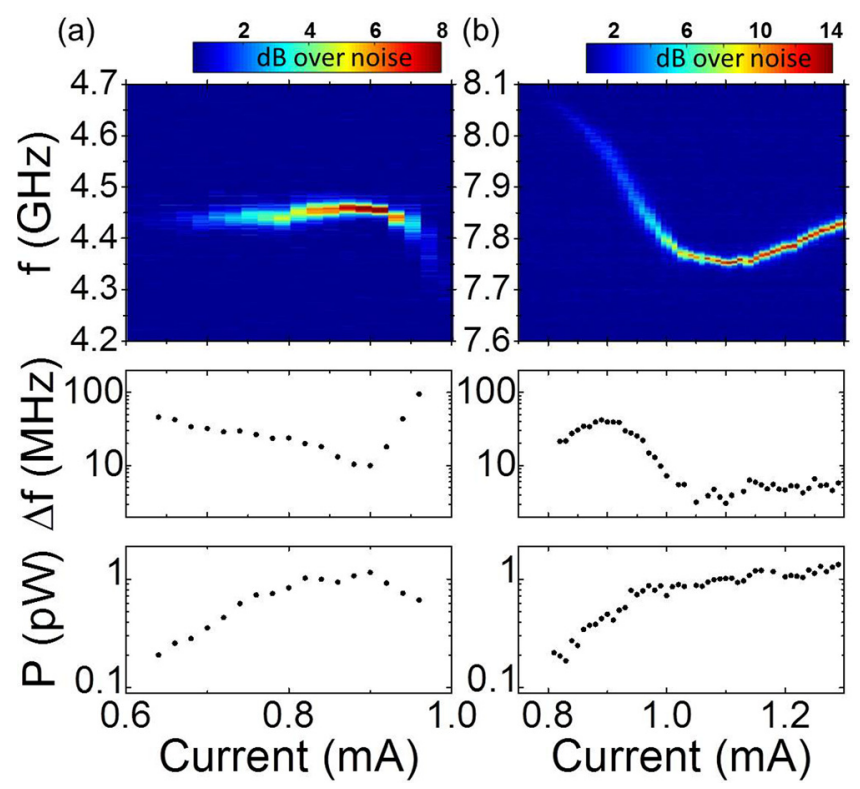

FIG. 4. Power spectral density (PSD), extracted linewidth, and integrated power of the auto-oscillations for (a) in-plane, and (b) out-of-plane magnetic fields.

linewidth, $\Delta H$ (white dots) as a function of frequency, together with a linear $\mathrm{fit}^{33} \Delta H=\Delta H_{0}+4 \pi \alpha f / \gamma$ (red line), from which an inhomogeneous broadening $\left(\Delta H_{0}\right)$ of 1.8 Oe and a Gilbert damping $(\alpha)$ of $8.32 \times 10^{-3}$ can be extracted.

To extract the spin Hall angle $\theta_{S H}$, we then investigated the current-induced linewidth changes originating from the SOT of the W layer. Figure 3(b) shows how the linewidth depends linearly on the device current, with positive current providing a negative damping when the field is along $\phi=$ $30^{\circ}$ (black circle), and positive damping when the field direction is reversed to $210^{\circ}$ (red circle). The spin Hall angle, defined as the ratio of the spin and charge current densities $\theta_{S H}=J_{s} / J_{c}$, is then extracted from the slopes in Figure 3(b) using the following equation: $9,11,34$

$$
\theta_{S H}=\frac{\delta \Delta H / \delta I_{D C}}{\frac{2 \pi f}{\gamma} \frac{\sin \phi}{\left(H_{I P}+0.5 M_{e f f}\right) \mu_{0} M_{s} t} \frac{\hbar}{2 e}} \frac{R_{P y}+R_{W}}{R_{P y}} A_{C},
$$

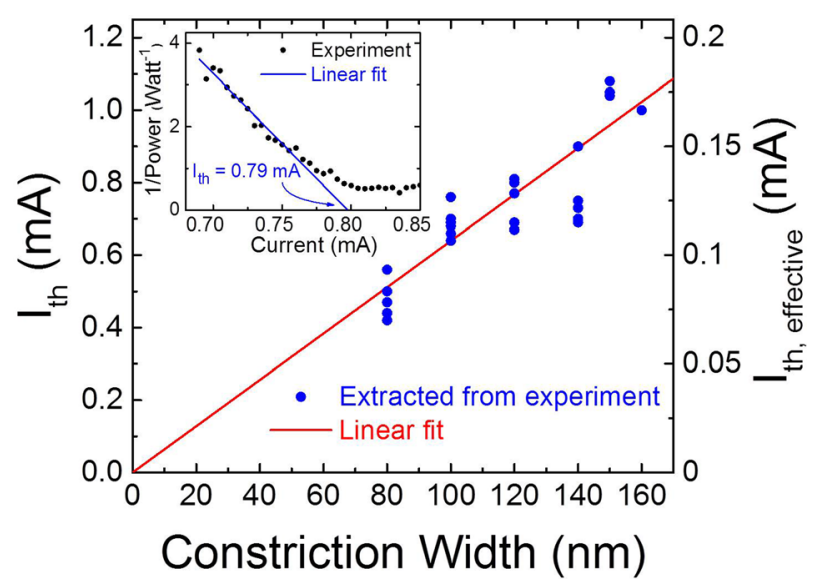

FIG. 5. Threshold current vs. constriction width in an 800 Oe in-plane magnetic field at $\phi=20^{\circ}$. Inset: threshold current extraction through a linear fit of 1 /power versus current at low current. where $M_{s}$ is the saturation magnetization, and $t$ is the thickness of the magnetic layer, $e$ is the electron charge and $\hbar$ is Planck's constant, $R_{N i F e}$ and $R_{W}$ are the resistances of the $\mathrm{NiFe}$ and tungsten layers, and $A_{C}$ is the cross-sectional area of the measured device. We obtained $\theta_{S H}=-0.385 \pm 0.009$, which is comparable to previous studies. ${ }^{10,21}$

We now turn to the SHNOs. Figure 4(a) shows the measured power spectral density (PSD) vs. SHNO drive current from a $140 \mathrm{~nm}$ wide nano-constriction in a field of $H_{I P}=400$ Oe along $\phi=20^{\circ}$. The auto-oscillation frequency, linewidth, and integrated power are extracted by fitting the peaks of all spectra with a Lorentzian function. Whereas the auto-oscillation frequency only depends weakly on the drive current, both the linewidth and the integrated power shows a rapid exponential improvement up until $I_{D C}=-0.9 \mathrm{~mA}$, reaching a minimum linewidth of about $10 \mathrm{MHz}$, and a maximum power of about $1 \mathrm{pW}$. Above this current level, the auto-oscillation frequency decreases, and both linewidth and power deteriorate, possibly indicating the appearance of a different mode. ${ }^{18,35,36}$

When the applied field is tilted out-of-plane $\left(\theta=80^{\circ}\right.$; $\left.\phi=20^{\circ}\right)$ and increased to $8 \mathrm{kOe}$ to align the Py magnetization (Figure 4(b)), the current dependence changes character to a much more non-monotonic frequency, with an initial red shift at low current, which changes to a clear blue shift at higher current. In contrast to the in-plane case, the integrated power shows a monotonic improvement with current, and the linewidth improves to about $2 \mathrm{MHz}$, i.e., close to an order of magnitude better than in the in-plane case. As shown in Ref. 20 this non-monotonic frequency behavior results from a gradual change in the location of the auto-oscillations. At low currents, the auto-oscillations start from the edges of the nano-constrictions. With increasing current, the autooscillation region then expands into the nano-constriction, and the point of maximum spin wave intensity moves inward. In this process, the mode center experiences a varying field landscape with the net effect being a frequency redshift as the mode leaves the edges followed by a blue-shift as it further expands inward.

The large spin Hall angle is expected to have a strong beneficial impact on the threshold current $\left(I_{t h}\right)$ for microwave signal generation. To evaluate this, we measured more than 20 SHNOs with different nano-constriction widths ranging from 80 to $160 \mathrm{~nm}$. $I_{\text {th }}$ is determined from linear fits of the inverse microwave power vs. current, ${ }^{37,38}$ as shown in the inset of Figure 5; all the measurements were carried out with $H_{I P}=0.08 \mathrm{~T}$ and $\phi=20^{\circ}$. Figure 5 shows that $I_{t h}$ depends linearly on the nano-constriction width, and the values observed are more than $60 \%$ lower than those from previous reports on Pt-based SHNOs. $2,13,14,16-18,21,23$

The effective threshold current flowing through the W layer, shown on the right-hand y-axis of Figure 5, can be calculated by considering the ratio of the Py and $\mathrm{W}$ resistivities. This shows that a current of only about $75 \mu \mathrm{A}$ in $\mathrm{W}$ can excite Py auto-oscillations in a $80 \mathrm{~nm}$ nano-constriction. From a linear fit to all the measured devices, we can calculate the effective threshold current density in our W-based SHNOs to be $J_{\text {th }, \text { eff }} \simeq 2 \times 10^{7} \mathrm{~A} / \mathrm{cm}^{2}$.

We have demonstrated a reliable fabrication and operation of W/Py based nano-constriction SHNOs. Our devices 
achieved a significant reduction in the threshold current of more than $60 \%$ compared to Pt/Py devices. Our findings thus lay out a definite development path for low-current SHNOs using highly efficient spin orbit torque from a W layer.

This work was supported by the Swedish Foundation for Strategic Research (SSF), the Swedish Research Council (VR), and the Knut and Alice Wallenberg foundation (KAW). This work was also supported by the European Research Council (ERC) under the European Community's Seventh Framework Programme (FP/2007-2013)/ERC Grant 307144 "MUSTANG."

${ }^{1}$ T. Chen, R. K. Dumas, A. Eklund, P. K. Muduli, A. Houshang, A. A. Awad, P. Dürrenfeld, B. G. Malm, A. Rusu, and J. Åkerman, Proc. IEEE 104, 1919 (2016).

${ }^{2}$ V. E. Demidov, S. Urazhdin, H. Ulrichs, V. Tiberkevich, A. Slavin, D. Baither, G. Schmitz, and S. O. Demokritov, Nat. Mater. 11, 1028 (2012).

${ }^{3}$ J. E. Hirsch, Phys. Rev. Lett. 83, 1834 (1999).

${ }^{4}$ S. Zhang, Phys. Rev. Lett. 85, 393 (2000).

${ }^{5}$ Y. K. Kato, R. C. Myers, A. C. Gossard, and D. D. Awschalom, Science 306, 1910 (2004).

${ }^{6}$ J. Wunderlich, B. Kaestner, J. Sinova, and T. Jungwirth, Phys. Rev. Lett. 94, 047204 (2005).

${ }^{7}$ E. Saitoh, M. Ueda, H. Miyajima, and G. Tatara, Appl. Phys. Lett. 88, 182509 (2006).

${ }^{8}$ S. O. Valenzuela and M. Tinkham, Nature 442, 176 (2006).

${ }^{9}$ L. Liu, T. Moriyama, D. Ralph, and R. Buhrman, Phys. Rev. Lett. 106, 036601 (2011).

${ }^{10}$ C.-f. Pai, L. Liu, Y. Li, H. W. Tseng, D. C. Ralph, and R. A. Buhrman, Appl. Phys. Lett. 101, 122404 (2012).

${ }^{11}$ K.-U. Demasius, T. Phung, W. Zhang, B. P. Hughes, S.-H. Yang, A. Kellock, W. Han, A. Pushp, and S. S. P. Parkin, Nat. Commun. 7, 10644 (2016).

${ }^{12}$ L. Liu, C.-F. Pai, Y. Li, H. W. Tseng, D. C. Ralph, and R. A. Buhrman, Science 336, 555 (2012).

${ }^{13}$ R. H. Liu, W. L. Lim, and S. Urazhdin, Phys. Rev. Lett. 110, 147601 (2013).

${ }^{14}$ H. Ulrichs, V. E. Demidov, S. O. Demokritov, W. L. Lim, J. Melander, N. Ebrahim-Zadeh, and S. Urazhdin, Appl. Phys. Lett. 102, 132402 (2013).

${ }^{15}$ V. E. Demidov, H. Ulrichs, S. V. Gurevich, S. O. Demokritov, V. S. Tiberkevich, A. N. Slavin, A. Zholud, and S. Urazhdin, Nat. Commun. 5, 3179 (2014).

${ }^{16}$ V. E. Demidov, S. Urazhdin, A. Zholud, A. V. Sadovnikov, and S. O. Demokritov, Appl. Phys. Lett. 105, 172410 (2014).

${ }^{17}$ A. Zholud and S. Urazhdin, Appl. Phys. Lett. 105, 112404 (2014).
${ }^{18}$ Z. Duan, A. Smith, L. Yang, B. Youngblood, J. Lindner, V. E. Demidov, S. O. Demokritov, and I. N. Krivorotov, Nat. Commun. 5, 5616 (2014).

${ }^{19}$ L. Yang, R. Verba, V. Tiberkevich, T. Schneider, A. Smith, Z. Duan, B. Youngblood, K. Lenz, J. Lindner, A. N. Slavin, and I. N. Krivorotov, Sci. Rep. 5, 16942 (2015).

${ }^{20}$ A. A. Awad, P. Dürrenfeld, A. Houshang, M. Dvornik, E. Iacocca, R. K. Dumas, and J. Akerman, "Long-range mutual synchronization of spin Hall nano-oscillator," Nat. Phys. (published online 2016).

${ }^{21}$ M. Collet, X. de Milly, O. d'Allivy Kelly, V. V. Naletov, R. Bernard, P. Bortolotti, J. Ben Youssef, V. E. Demidov, S. O. Demokritov, J. L. Prieto, M. Muñoz, V. Cros, A. Anane, G. de Loubens, and O. Klein, Nat. Commun. 7, 10377 (2016).

${ }^{22}$ A. Hamadeh, O. d'Allivy Kelly, C. Hahn, H. Meley, R. Bernard, A. H. Molpeceres, V. V. Naletov, M. Viret, A. Anane, V. Cros, S. O. Demokritov, J. L. Prieto, M. Muñoz, G. de Loubens, and O. Klein, Phys. Rev. Lett. 113, 197203 (2014).

${ }^{23}$ M. Ranjbar, P. Durrenfeld, M. Haidar, E. Iacocca, M. Balinskiy, T. Q. Le, M. Fazlali, A. Houshang, A. A. Awad, R. K. Dumas, and J. Akerman, IEEE Magn. Lett. 5, 3000504 (2014).

${ }^{24}$ Y. Niimi, M. Morota, D. H. Wei, C. Deranlot, M. Basletic, A. Hamzic, A. Fert, and Y. Otani, Phys. Rev. Lett. 106, 126601 (2011).

${ }^{25}$ M. Yamanouchi, L. Chen, J. Kim, M. Hayashi, H. Sato, S. Fukami, S. Ikeda, F. Matsukura, and H. Ohno, Appl. Phys. Lett. 102, 212408 (2013).

${ }^{26}$ Y. Niimi, Y. Kawanishi, D. H. Wei, C. Deranlot, H. X. Yang, M. Chshiev, T. Valet, A. Fert, and Y. Otani, Phys. Rev. Lett. 109, 156602 (2012).

${ }^{27}$ Q. Hao, W. Chen, and G. Xiao, Appl. Phys. Lett. 106, 182403 (2015).

${ }^{28}$ E. Lassner and W. D. Schubert, Tungsten: Properties, Chemistry, Technology of the Element, Alloys, and Chemical Compounds (Springer US, 2012).

${ }^{29}$ J. Sankey, P. Braganca, A. Garcia, I. Krivorotov, R. Buhrman, and D. Ralph, Phys. Rev. Lett. 96, 227601 (2006).

${ }^{30}$ J. C. Sankey, Y.-T. Cui, J. Z. Sun, J. C. Slonczewski, R. a. Buhrman, and D. C. Ralph, Nat. Phys. 4, 67 (2007).

${ }^{31}$ W. Chen, G. de Loubens, J.-M. L. Beaujour, J. Z. Sun, and A. D. Kent, Appl. Phys. Lett. 95, 172513 (2009).

${ }^{32}$ X. Cheng, J. A. Katine, G. E. Rowlands, and I. N. Krivorotov, Appl. Phys. Lett. 103, 082402 (2013).

${ }^{33}$ C. Kittel, "On the theory of ferromagnetic resonance absorption," Phys. Rev. 73, 155 (1948).

${ }^{34}$ K. Ando, S. Takahashi, K. Harii, K. Sasage, J. Ieda, S. Maekawa, and E. Saitoh, Phys. Rev. Lett. 101, 036601 (2008).

${ }^{35}$ A. Giordano, M. Carpentieri, A. Laudani, G. Gubbiotti, B. Azzerboni, and G. Finocchio, Appl. Phys. Lett. 105, 042412 (2014).

${ }^{36}$ H. Ulrichs, V. E. Demidov, and S. O. Demokritov, Appl. Phys. Lett. 104, 042407 (2014).

${ }^{37}$ V. Tiberkevich, A. Slavin, and J.-V. Kim, Appl. Phys. Lett. 91, 192506 (2007).

${ }^{38}$ A. Slavin and V. Tiberkevich, IEEE Trans. Magn. 45, 1875 (2009). 